\title{
TEXTURE EVOLUTION IN A HOT ROLLED AUSTENITIC STAINLESS STEEL
}

\author{
C. D. SINGH, ${ }^{1}$ V. RAMASWAMY ${ }^{1}$ and C. SURYANARAYANA ${ }^{2}$
}
${ }^{1}$ Research and Development Centre for Iron and Steel, Steel Authority of India Limited, Ranchi - 834002, India.
${ }^{2}$ Department of Metallurgical Engineering, Banaras Hindu University, Varanasi-221005, India

(Received August 1, 1990)

The ODF analysis of the surface texture of the hot band of austenitic stainless steel reveals the presence of orientations of shear texture. These orientation elements are mainly distributed along two limited tubes of preferred orientations. The fibre of the first tube has its axis $\langle 110\rangle \| R D$ and runs from $\{001\}\langle 110\rangle$ to an orientation near $\{112\}\langle 110\rangle$ whereas the fibre of the second tube is inclined $30^{\circ}$ from ND towards RD (i.e. $\langle 110\rangle 30^{\circ} \mathrm{ND}$ fibre) and stretches from $\{111\}\langle 112\rangle+5^{\circ}$ to the orientation near $\{112\}\langle 110\rangle$. The shear texture components are formed at the surface by shearing during hot rolling. Once they are formed, they get partly recrystallized by dynamic in situ recrystallization. On the other hand a duplex texture (i.e. retained copper type and recrystallization type) is present at the centre level of the hot band. The orientation elements of copper type (i.e. $\langle 110\rangle 60^{\circ} \mathrm{ND}$ fibre) are $\{011\}\langle 112\rangle, \approx\{123\}\langle 634\rangle$ and $\{112\}\langle 111\rangle$ whereas the recrystallization texture components are dominated by cube $\{001\}\langle 100\rangle$ orientation and other RD rotated cubes through oriented nucleation during dynamic recrystallization.

KEY WORDS Shear texture, rolling texture, recrystallization texture, dynamic recrystallization, oriented nucleation, oriented growth.

\section{INTRODUCTION}

During hot rolling of metals, the surface undergoes a shear-strain deformation whereas the interior layers are subjected to plain-strain deformation. Depending on the conditions of rolling i.e. plain-strain or shear-strain, one obtains the rolling or shear textures in deformed metals. High surface friction, among other variables, favours the formation of shear textures near the surface of the rolled sheets (Regenet and Stüwe, 1963, Dillamore and Roberts, 1963/64, Truszkowski, Krol and Major, 1980). In face centred cubic metals and alloys, the shear textures have been described either in terms of ideal orientations $\{001\}\langle 110\rangle$, $\{112\}\langle 110\rangle,\{111\}\langle 110\rangle$ and $\{111\}\langle 112\rangle$ or by the spread range between these orientations (Backofen, 1950, Backofen and Hundy, 1953, Williams, 1962). The spread range has also been described in terms of an orientation $\{001\}\langle 110\rangle$ and a complete fibre component $\{111\}$ parallel to shear plane (Hibbard, 1951, Williams, 1962, Regenet and Stüwe, 1963). Recently Smith (1978) investigated the surface texture of a commercially produced hot band of austenitic stainless steel and observed a combination of orientations i.e. $\{112\}\langle 110\rangle$ as major and $\{111\}\langle 110\rangle$ as minor to characterize the surface texture. Besides experimental 
observations, attempts have been made to determine the shear textures theoretically from the calculations based on Taylor theory (Taylor, 1938) under plain-strain condition (Dillamore and Katoh, 1974a) and under shear-strain condition (Hansen and Mecking, 1975). In addition, the shear textures have been calculated from Taylor theory adapted to both slip and twinning (Gill Sevillano, Van Houtte and Aernoudt, 1975, Van Houtte and Aernoudt, 1975, Van Houtte and Aernoudt, 1976, Van Houtte, 1978).

The rolling textures of face centred cubic metals and alloys possess different characters (i.e. brass type and copper type) depending on the rolling degree, rolling temperature and the stacking fault energy (SFE) and have been described in terms of two limited fibres i.e. a fibre axis $\langle 110\rangle \| N D$ extending from $\{011\}\langle 100\rangle$ to $\{011\}\langle 112\rangle$ and the other fibre $\langle 110\rangle 60^{\circ} \mathrm{ND}$ running from $\{112\}\langle 111\rangle$ through $\{123\}\langle 634\rangle$ to $\{011\}\langle 112\rangle$ with $\langle 110\rangle$ axis inclined about $60^{\circ}$ from ND towards RD (Hirsch and Lücke, 1988). While the brass type (low SFE) is characterized by $\langle 110\rangle \| N D$ fibre with $\{011\}\langle 112\rangle$ as major orientation, the copper type rolling texture forms in intermediate to high SFE metals and alloys and is described mainly by $\langle 110\rangle 60^{\circ} \mathrm{ND}$ fibre. The transition texture in between copper type and brass type is also perceptible. The texture transition from brass to copper type has been systematically surveyed as a function of rolling temperature in austenitic stainless steel (Goodman and Hu, 1964). Further, it has been reported by Lücke (1954) that the copper type texture is not affected much by raising the rolling temperature until the temperature is sufficiently high for recrystallization to occur. At and above such temperatures a mixture of rolling and recrystallization textures (i.e. duplex texture) forms. The well known cube texture $\{001\}\langle 100\rangle$ forming from copper type rolling texture on recrystallization has been used along with the $\{021\}\langle 100\rangle$ component to represent the texture at the centre of the hot band of austenitic stainless steel (Smith, 1978).

The texture characterization of the hot band of austenitic stainless steel was based on the pole figure analysis (Smith, 1978) which possesses very limited resolving power in revealing the differences which are more of quantitative than of qualitative nature. For a complete, exact and explicit description of texture, the orientation distribution function (ODF) is required, which can be obtained by the mathematical procedure of pole-figure inversion (Bunge, 1969). This paper concerns with the results of the ODF analysis of the texture near the surface and at the centre of the hot band of a commercially produced austenitic stainless steel and also discusses the process of texture evolution during hot rolling.

\section{EXPERIMENTAL PROCEDURE}

A hot band (HB) of an austenitic stainless steel of nominal composition (by wt\%): $19.25 \% \mathrm{Cr}, 8.40 \% \mathrm{Ni}, 0.05 \% \mathrm{C}, 1.24 \% \mathrm{Mn}, 0.48 \% \mathrm{Si}, 0.02 \% \mathrm{P}, 0.02 \% \mathrm{~S}$ was commercially produced in the hot strip mill and used for the present investigation. As per supplier's information $2.95 \mathrm{~mm}$ thick HB strip was finished at about $1173 \mathrm{~K}$ and coiled at about $1023 \mathrm{~K}$. The hot band was pickled in a pickling solution (by volume \%) of $10 \% \mathrm{HNO}_{3}+2 \% \mathrm{HF}+88 \%$ distilled water at a temperature $323 \mathrm{~K}$ for a period of 5 minutes to remove any oxides and scales on its surface. 
Optical metallography was performed on specimens mechanically polished and electrolytically etched in saturated oxalic acid solution at 10 volts for 2 minutes at sections $(S=1, S=0)$ cut perpendicular to the transverse direction containing rolling (R) and normal (N) direction RN. Here $S$ denotes the thickness level at which the metallography was done and is defined as the ratio of the distance from the specimen centre to the level to the half thickness. The hardness of the HB was measured for $S=1$ and $S=0$ levels at the sections cut parallel to the rolling plane using $5 \mathrm{~kg}$ load on standard Vickers hardness tester.

$\mathrm{X}$-ray texture measurements were carried out at the sections (i.e. $S=1$ and $S=0$ levels) parallel to the strip surface by back reflection technique using $\mathrm{MOK}_{\alpha}$ radiation (Schulz, 1949). For each ground and etched specimen $(20 \mathrm{~mm} \times 14 \mathrm{~mm})$ the texture was determined by measuring four incomplete pole figures (maximum tilt angle $\left.75^{\circ}\right)$ of the plane $\{111\},\{200\},\{220\}$ and $\{113\}$ and plotting the ODF. The measured intensity was subjected to background, geometrical and defocussing corrections using a random specimen of pressed and sintered austenitic stainless steel powders. The ODF was calculated from the data of four incomplete pole figures following the series expansion method of Bunge (1969) and using the pseudo-normalization technique of Kern and Bergmann (1978). The series was extended up to $l_{\max }=22$ using even series only.

\section{EXPERIMENTAL RESULTS}

The commercially produced hot band of austenitic stainless steel was fully austenitic. Figure 1(a) shows the optical micrograph of the HB in the RN section near the surface $(S=1)$. The contrast in the micrograph reveals that the outer layers are not fully recrystallized and both elongated and equiaxed grains, representative of deformation and dynamic recrystallization, are present. On the other hand, the microstructure of the central layers $(S=0)$ of the HB depicts mostly the elongated grains [Figure 1(b)] and thus the interior has retained the deformed structure. Some evidence of recrystallized grains is also present. The dark bands in the above micrographs appear to be thin sheet like bands of heavily deformed austenite grains. The average grain size measured on the sections parallel to the rolling plane near the surface and at the centre of the HB was about $7 \mu \mathrm{m}$ whereas the measured hardness values were about $235 \mathrm{HV}_{5}$ and $269 \mathrm{HV}_{5}$ respectively.

Figure 2 shows the three dimensional orientation density distribution (i.e. ODF) of crystallites for the surface $(S=1)$ of the HB in constant $\phi_{1}$ sections through Euler space. This ODF exhibits maxima which are presented in Table 1 along with their Euler angles. While the table gives the exact orientations of the maxima, in the text, approximate orientations are used as indicated by the symbol $\approx$ to represent them by simpler indices. The maximum orientation density is exhibited by the texture component $\{7712\}\langle 110\rangle$ which is about $5^{\circ}$ away from the ideal orientation $\{112\}\langle 110\rangle$ rotated about RD. The other texture components present on the surface of the $\mathrm{HB}$, in the decreasing order of density, are mainly $\{118\}\langle 110\rangle,\{332\}\langle 110\rangle(\approx\{111\}\langle 110\rangle),\{001\}\langle 110\rangle,\{232\}\langle 225\rangle$ $(\approx\{111\}\langle 112\rangle), \quad\{111\}\langle 112\rangle+5^{\circ}(\approx\{111\}\langle 112\rangle)$ and $\left\{\begin{array}{lll}9 & 12 & 8\end{array}\right\}\langle 3712\rangle$ $(\approx\{111\}\langle 123\rangle)$. Apart from these orientation elements, an orientation 


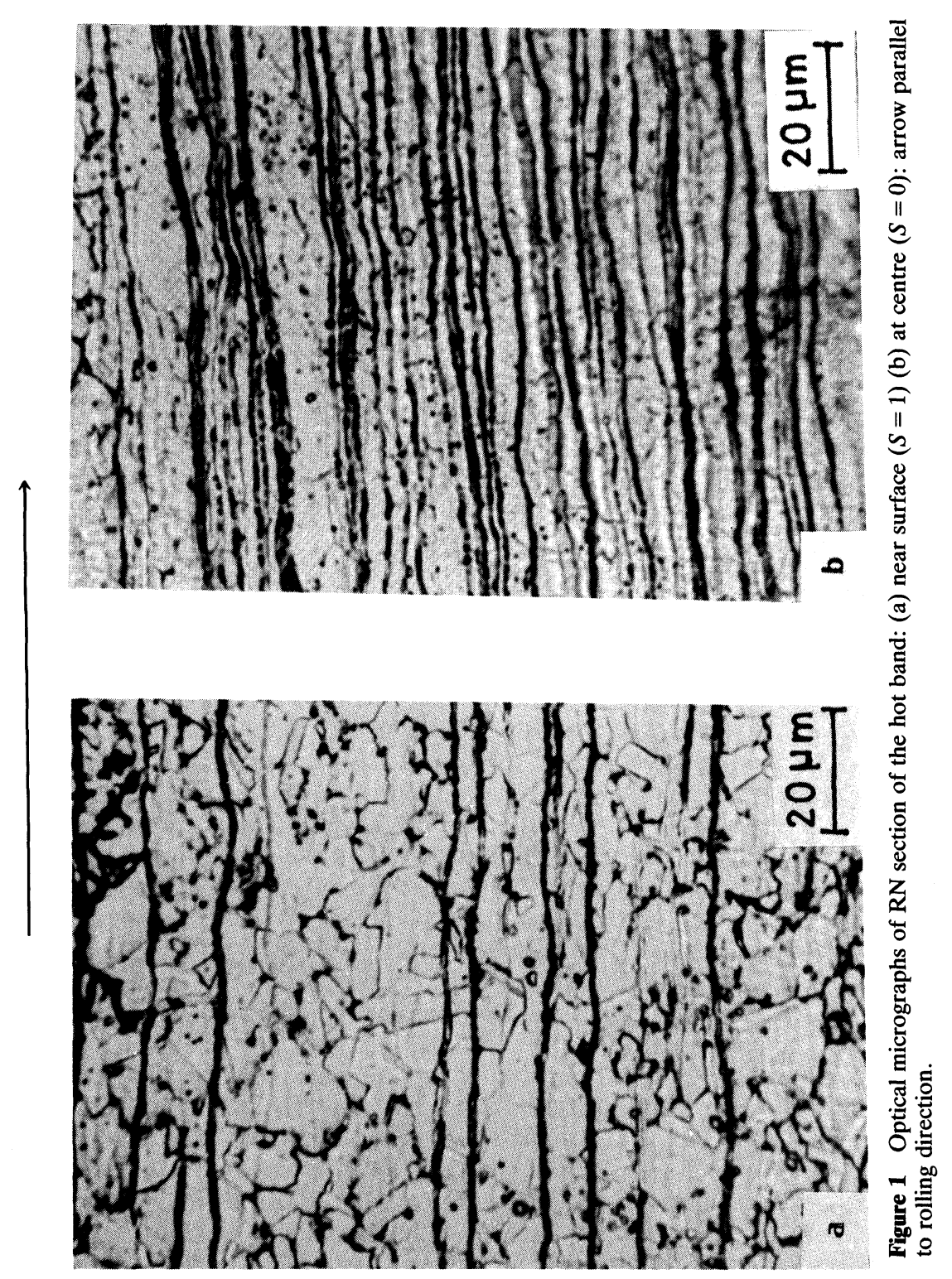



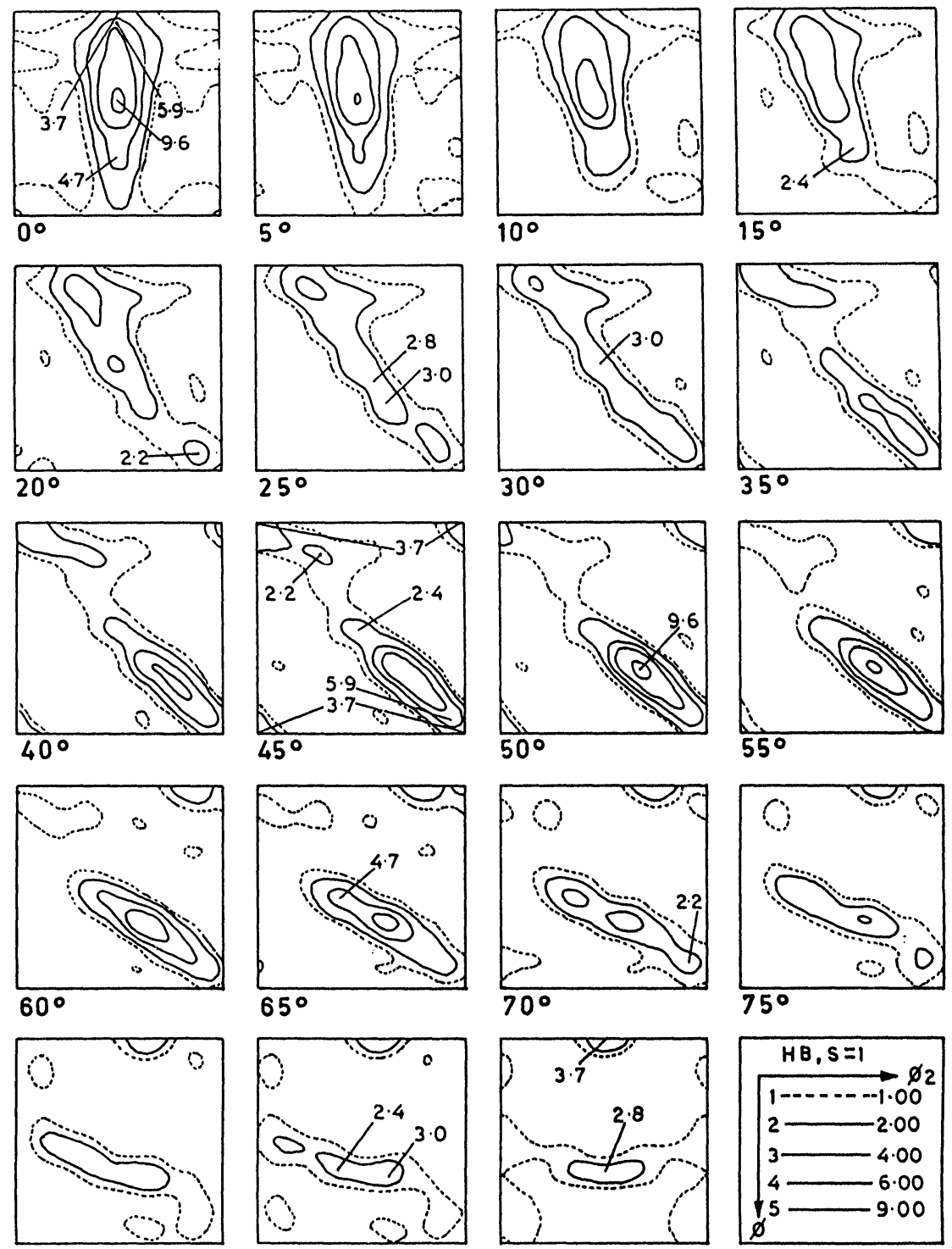

$65^{\circ}$

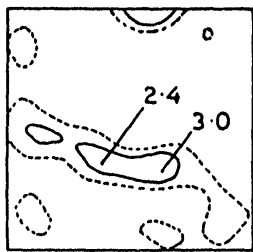

$85^{\circ}$
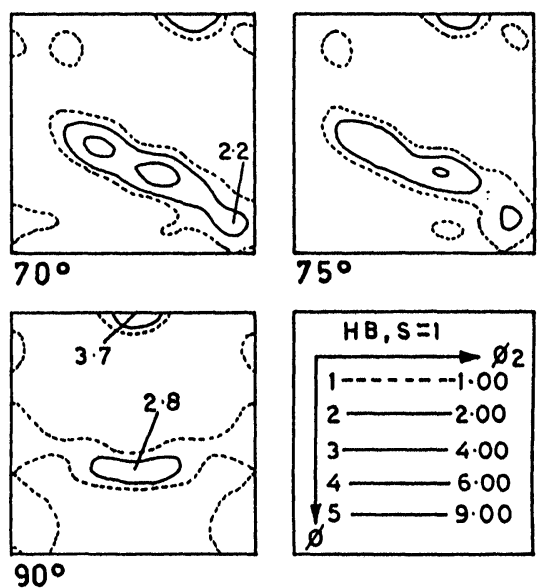

Figure 2 ODF for $S=1$ level of hot band (HB) in constant $\phi_{1}$ sections.

$\{129\}\langle 4112\rangle$ which is nearer to $\{139\}\langle 341\rangle$ appears on the surface as a minor component. Most of the prominent orientations of the surface texture of the HB are contained in $\phi_{2}=45^{\circ}$ section (Figure 3) which is chosen here as a concise though not complete representation of the texture components. This figure depicts clearly the two limited tubes of preferred orientations in the orientation space. One of the orientation tubes has its $\langle 110\rangle$ fibre axis parallel to RD and 
Table 1 Orientation density $f(g)$ of the texture components $\{H K L\}\langle U V W\rangle$ near the surface $(S=1)$ of the hot band

\begin{tabular}{|c|c|c|c|c|c|}
\hline \multirow[t]{2}{*}{$H K L$} & \multirow[t]{2}{*}{$U V W$} & \multicolumn{3}{|c|}{ Euler angles } & \multirow{2}{*}{$\begin{array}{l}\text { Orientation density } \\
f(g)\end{array}$} \\
\hline & & $\phi_{1}$ & $\phi$ & $\phi_{2}$ & \\
\hline$\{001\}$ & $\langle 110\rangle$ & 0 & $\mathbf{0}$ & 45 & 3.7 \\
\hline$\{118\}$ & $\langle 110\rangle$ & 0 & 10 & 45 & 5.9 \\
\hline $\begin{array}{l}\left\{\begin{array}{ll}7 & 12\end{array}\right\} \\
\text { (equal to) }\end{array}$ & $\langle 110\rangle$ & $\mathbf{0}$ & 40 & 45 & 9.6 \\
\hline $\begin{array}{l}\{112\} \\
\quad\left(\text { rotated } 5^{\circ} \mathrm{RD}\right)\end{array}$ & $\langle 110\rangle$ & 0 & 35 & 45 & \\
\hline $\begin{array}{l}\{332\} \\
\quad(\text { equal to })\end{array}$ & $\langle 110\rangle$ & $\mathbf{0}$ & 65 & 45 & 4.7 \\
\hline $\begin{array}{l}\{111\} \\
\quad\left(\text { rotated } 10^{\circ} \mathrm{RD}\right)\end{array}$ & $\langle 110\rangle$ & 0 & 55 & 45 & \\
\hline $\begin{array}{l}\{111\} \\
\quad \text { (equal to) }\end{array}$ & $\langle 112\rangle+5^{\circ}$ & 90 & 60 & 45 & 2.8 \\
\hline$\{111\}$ & $\langle 112\rangle$ & 90 & 55 & 45 & \\
\hline$\{232\}$ & $\langle 225\rangle$ & 84 & 61 & 56 & 3.0 \\
\hline 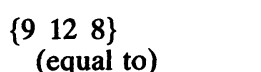 & $\left\langle\begin{array}{lll}3 & 7 & 12\end{array}\right\rangle$ & 84 & 58 & 34 & 2.4 \\
\hline$\{111\}$ & $\langle 123\rangle$ & 78 & 55 & 45 & \\
\hline $\begin{array}{l}\{129\} \\
\quad \text { (equal to) }\end{array}$ & $\left\langle\begin{array}{lll}4 & 11 & 2\end{array}\right\rangle$ & 44 & 14 & 27 & 2.2 \\
\hline$\{139\}$ & $\langle 341\rangle$ & 36 & 19 & 18 & \\
\hline
\end{tabular}

runs from an orientation $\{001\}\langle 110\rangle$ to $\approx\{112\}\langle 110\rangle$ with the maxima in orientation density observed at $\approx\{112\}\langle 110\rangle$ and the orientation distribution displaced towards $\approx\{111\}\langle 110\rangle$. The second tube has its $\langle 110\rangle$ fibre axis inclined about $30^{\circ}$ from ND towards RD (i.e. $\langle 110\rangle 30^{\circ} \mathrm{ND}$ fibre) and stretches from $\{111\}\langle 112\rangle+5^{\circ}$ to $\approx\{112\}\langle 110\rangle$. The orientation densities along the $\langle 110\rangle \| \mathrm{RD}$ fibre have been shown in Figure 4 which reveals clearly the profile of the peaks along the fibre. Besides a peak at $\{001\}\langle 110\rangle$ the profile contains a strong peak near $\{112\}\langle 110\rangle$ with two concealed peaks near $\{118\}\langle 110\rangle$ and $\{111\}\langle 110\rangle$. Figure 5 shows the orientation densities of the other texture components along
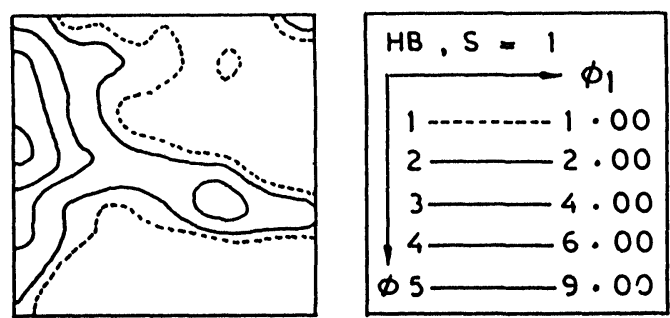

Figure 3 Section $\phi_{2}=45^{\circ}$ of the ODF for $S=1$ level of the hot band (HB). 


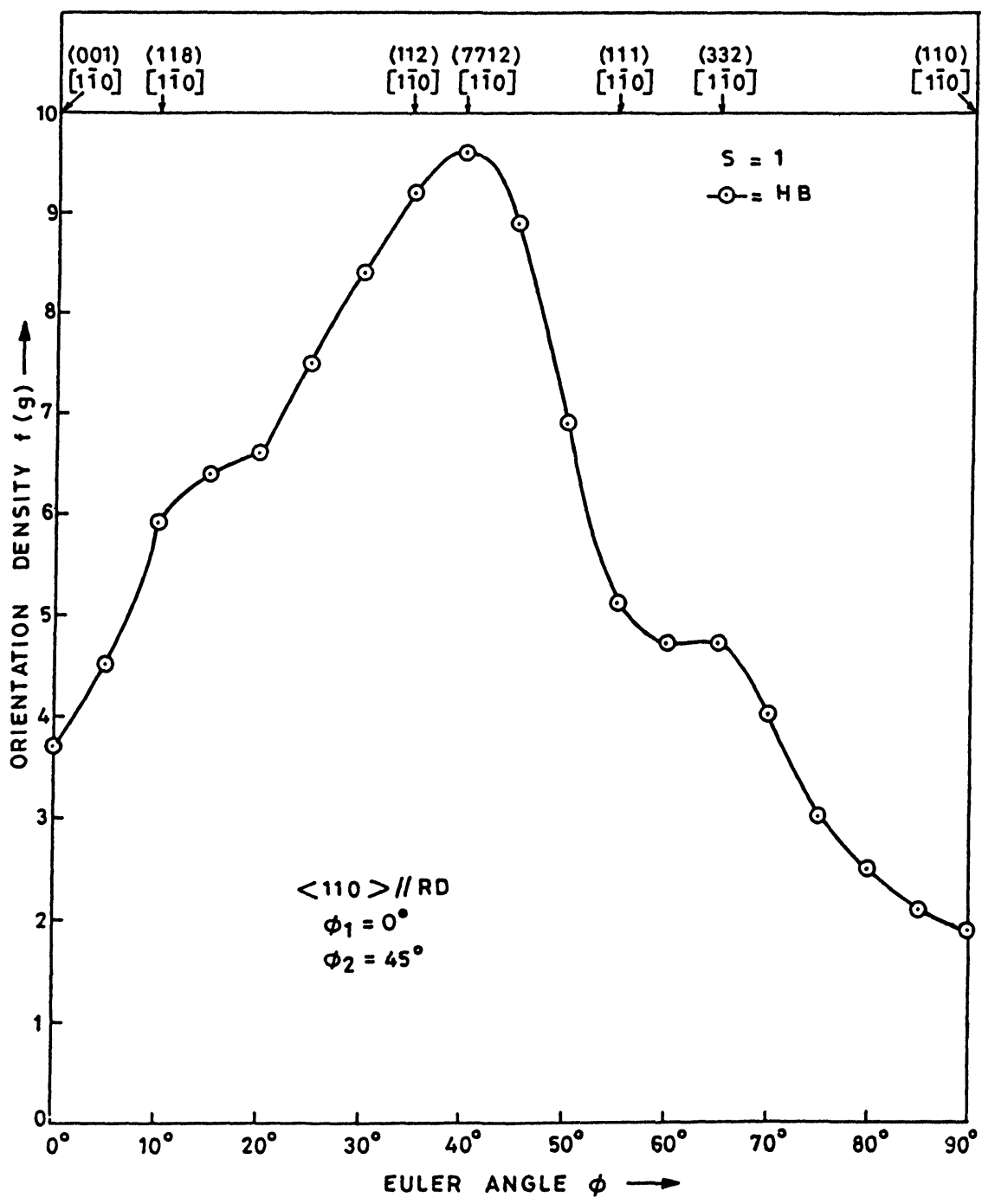

Figure 4 Orientation densities along the $\langle 110\rangle \| \mathrm{RD}$ fibre for the surface of the hot band (HB).

$\langle 110\rangle 30^{\circ} \mathrm{ND}$ fibre. It is evident from this figure that the density along the skeleton line increases strongly in the orientation $\approx\{112\}\langle 110\rangle$.

The ODF for the central layer $(S=0)$ of the HB in constant $\phi_{2}$ section is shown in Figure 6 and the maxima of orientations observed in this ODF along with their Euler angles are given in Table 2. The main texture components present in this ODF are mainly two types i.e. rolling (copper type) and recrystallization. The retained rolling texture components comprise of the orientation elements $\{011\}\langle 112\rangle, \approx\{123\}\langle 634\rangle$ and $\{112\}\langle 111\rangle$. The first of these is a major component and the others are minor. These elements are 


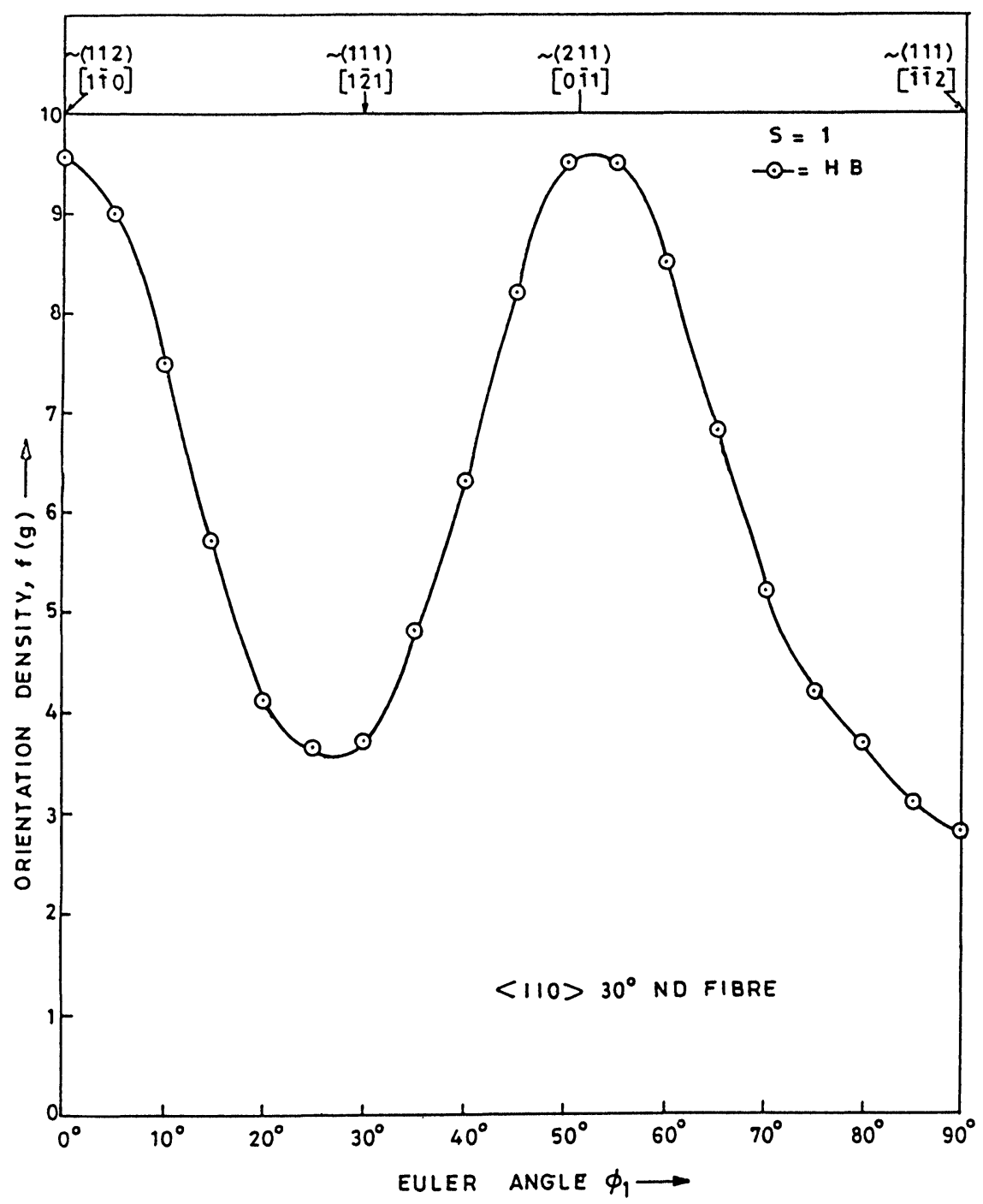

Figure 5 Orientation densities along the skeleton line of $\langle 110\rangle 30^{\circ} \mathrm{ND}$ fibre for the surface of the hot band (HB).

distributed along an orientation tube which is inclined to constant $\phi_{2}$ sections and runs from $\{011\}\langle 112\rangle$ at $\phi_{1}=35^{\circ}, \phi=45^{\circ}$ and $\phi_{2}=0^{\circ}$ to $\{112\}\langle 111\rangle$ at $\phi_{1}=39^{\circ}$, $\phi=66^{\circ}$, and $\phi_{2}=27^{\circ}$, or symmetrically equivalent and more convenient, from $\{112\}\langle 111\rangle$ at $\phi_{1}=90^{\circ}, \phi=35^{\circ}$ and $\phi_{2}=45^{\circ}$ to $\{011\}\langle 112\rangle$ at $\phi_{1}=35^{\circ}, \phi=45^{\circ}$ and $\phi_{2}=90^{\circ}$. The skeleton line of this orientation tube includes the orientation $\approx\{123\}\langle 634\rangle$ at $\phi_{1}=59^{\circ}, \phi=37^{\circ}$ and $\phi_{2}=63^{\circ}$ and has its $\langle 110\rangle$ fibre axis inclined $\sim 60^{\circ}$ from ND towards RD. The distribution of orientation densities along $\langle 110\rangle 60^{\circ} \mathrm{ND}$ fibre has been shown in Figure 7. This figure also indicates 

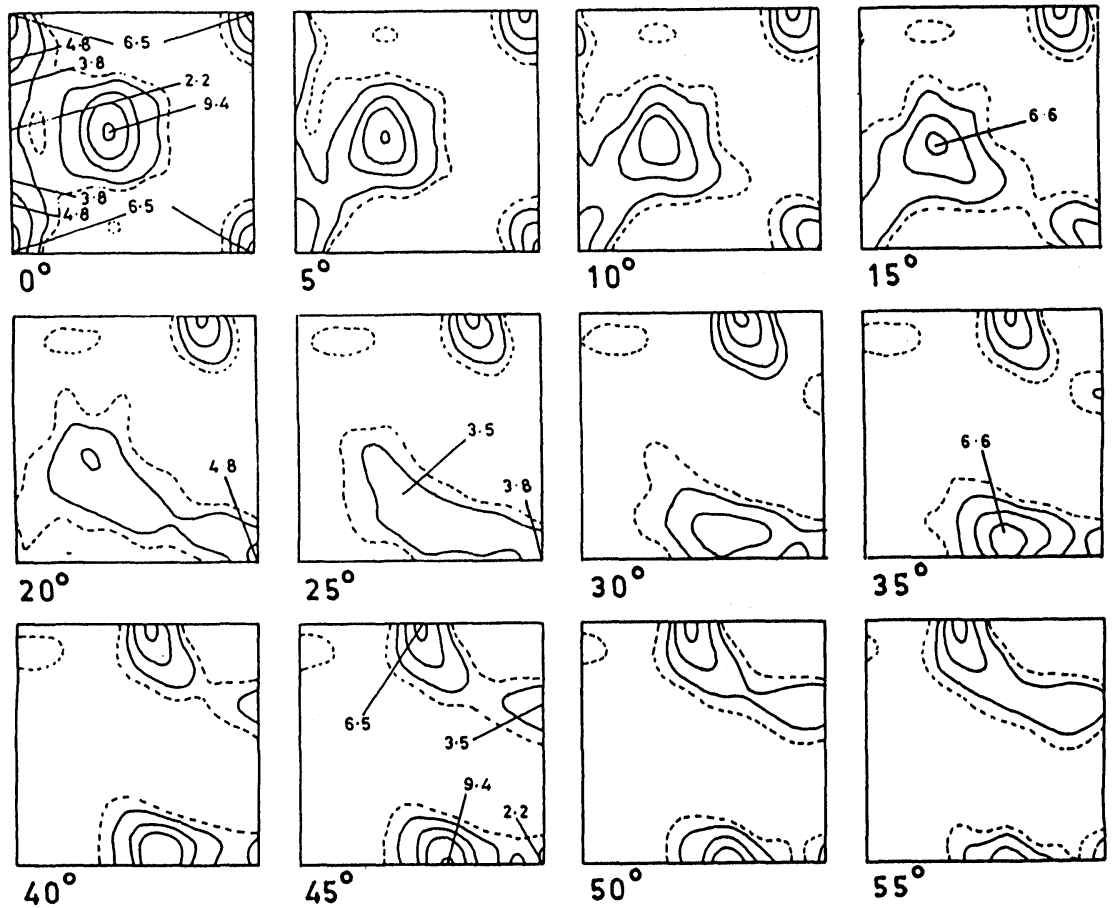

$25^{\circ}$
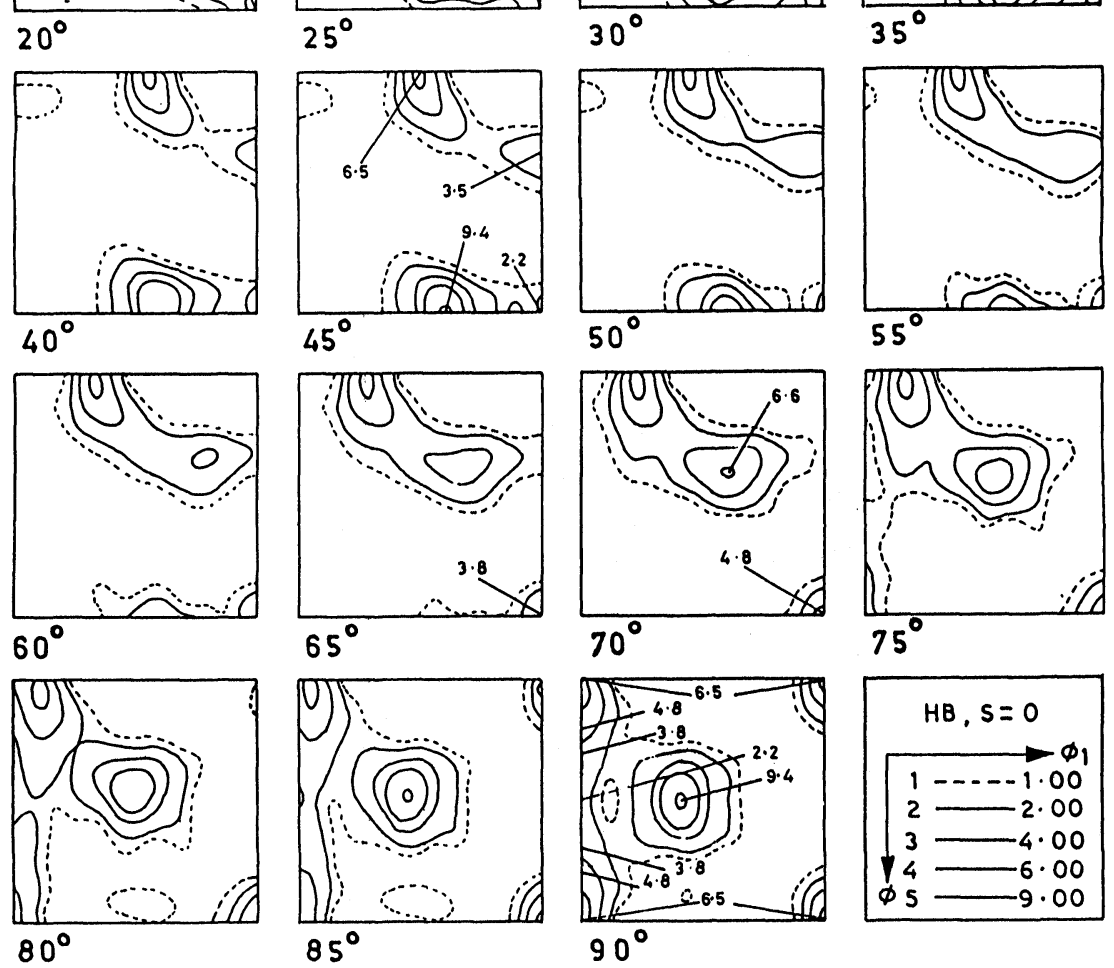

Figure 6 ODF for $S=0$ level of the hot band (HB) in constant $\phi_{2}$ sections.

clearly that the density is maximum at $\{011\}\langle 112\rangle$ and minimum at $\{112\}\langle 111\rangle$. On the other hand, the cube orientation $\{001\}\langle 100\rangle$ and the RD rotated cubes $\{013\}\langle 100\rangle,\{012\}\langle 100\rangle$ and Goss $\{011\}\langle 100\rangle$ constitute the recrystallization texture. The scattering of the cube orientation at $\phi_{1}=\phi=\phi_{2}=0^{\circ}$ towards $\{011\}\langle 100\rangle$ at $\phi_{1}=0^{\circ}, \phi=45^{\circ}$ and $\phi_{2}=0^{\circ}$ is clearly observed in Figure 6 while Figure 8 illustrates the density distribution of all the orientations in the zone 


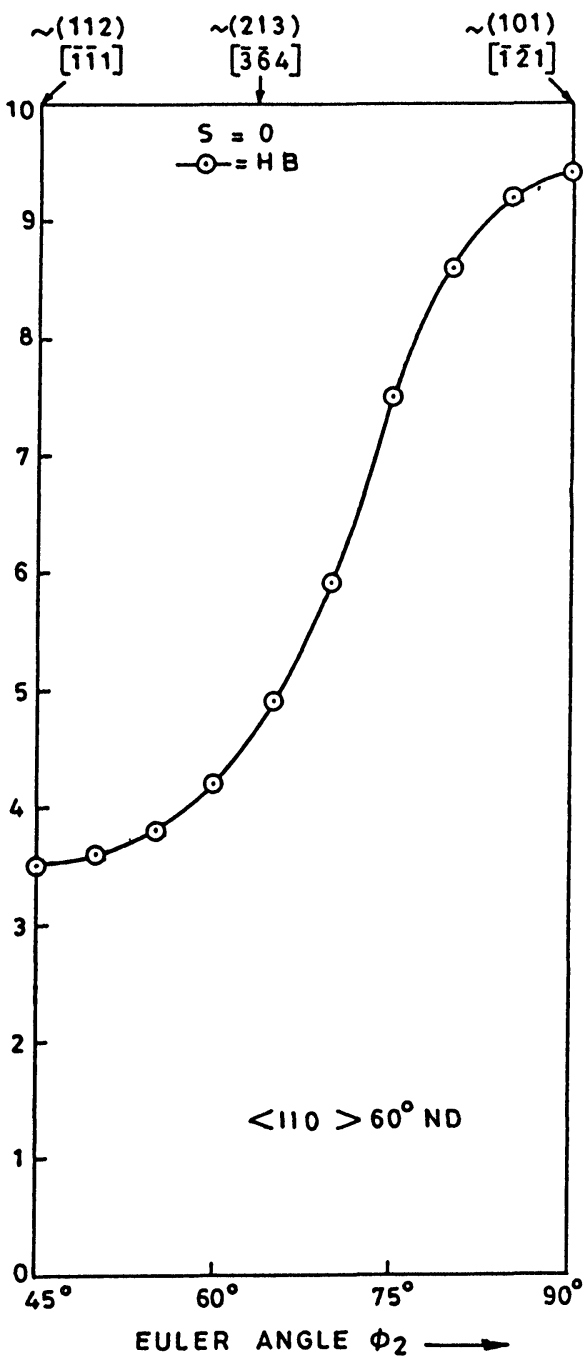

Figure 7 Orientation densities along the skeleton line of $\langle 110\rangle 60^{\circ} \mathrm{ND}$ fibre at the centre of the hot band (HB).

between the cube and $\{011\}\langle 100\rangle$ positions along $\langle 100\rangle \| \mathrm{RD}$ fibre for the constant $\phi_{2}=0^{\circ}$ section, where the maximum is observed at cube position and the minimum at $\{011\}\langle 100\rangle$ position.

\section{DISCUSSION}

The microstructures [Figure 1(a) and (b)] near the surface $(S=1)$ and at the centre $(S=0)$ of the HB reveal the presence of deformed as well as recrystallized grains. Further, the interior layers of the HB retain considerably a deformation structure. In addition, the average hardness value measured at central layers $\left(269 \mathrm{HV}_{5}\right)$ is larger than that near the surface $\left(235 \mathrm{HV}_{5}\right)$. All these evidences 


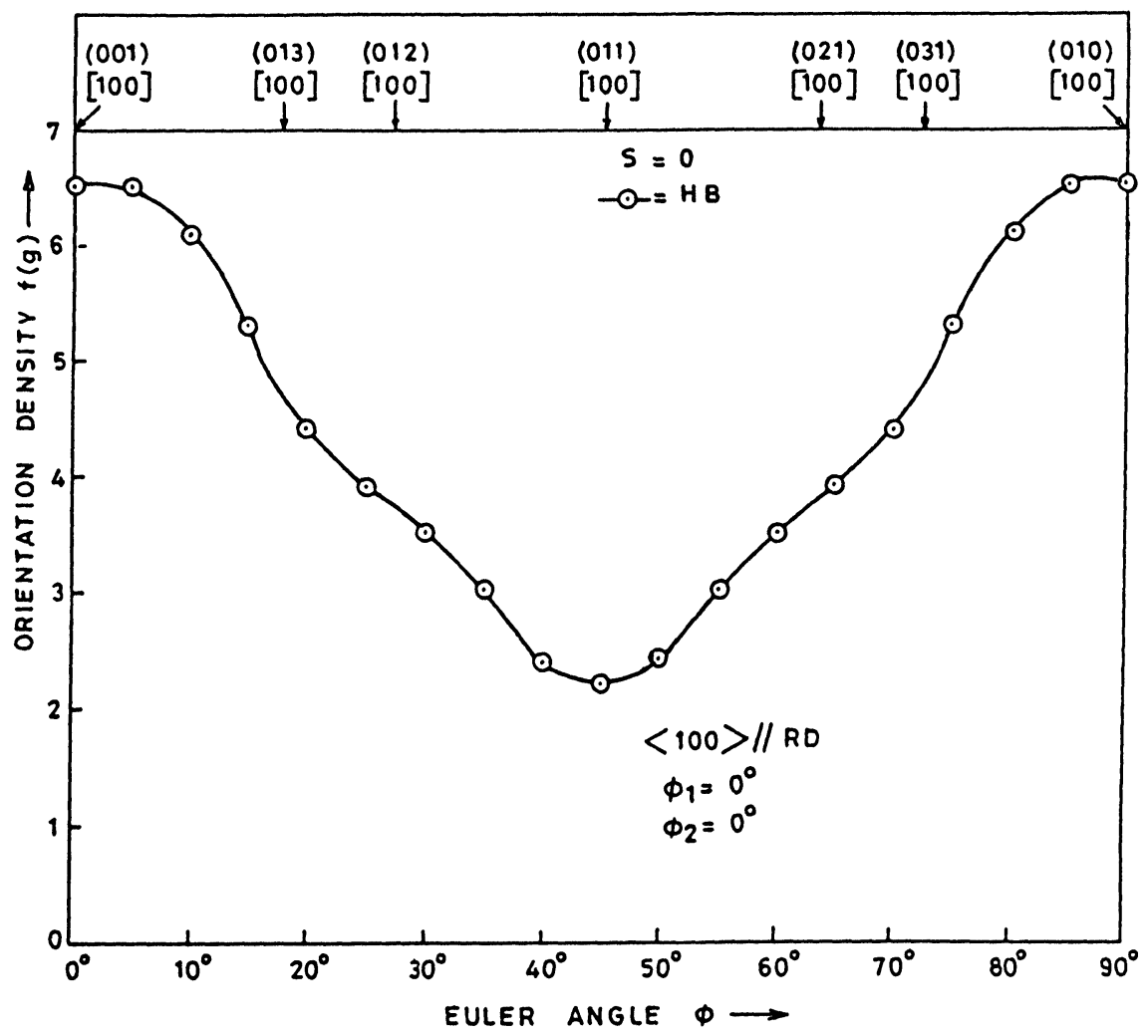

Figure 8 Orientation densities of the recrystallized texture components in the zone between cube $\{001\}\langle 100\rangle$ and Goss $\{011\}\langle 100\rangle$ positions along $\langle 100\rangle \| R D$, at the centre of the hot band (HB).

Table 2 Orientation density $f(g)$ of the texture components $\{\mathrm{HKL}\}\langle\mathrm{UVW}\rangle$ at the centre $(S=0)$ of the hot band

\begin{tabular}{|c|c|c|c|c|c|}
\hline \multirow[t]{2}{*}{$H K L$} & \multirow[t]{2}{*}{$U V W$} & \multicolumn{3}{|c|}{ Euler angles } & \multirow{2}{*}{$\begin{array}{l}\text { Orientation density } \\
f(g)\end{array}$} \\
\hline & & $\phi_{1}$ & $\phi$ & $\phi_{2}$ & \\
\hline \multicolumn{6}{|c|}{ Retained rolling texture } \\
\hline$\{011\}$ & $\langle 112\rangle$ & 35 & 45 & 0 & 9.4 \\
\hline $\begin{array}{l}\{134\} \\
\quad \text { (equal to) }\end{array}$ & $\langle 956\rangle$ & 54 & 38 & 72 & 6.6 \\
\hline$\{123\}$ & $\langle 634\rangle$ & 59 & 37 & 63 & \\
\hline$\{112\}$ & $\langle 111\rangle$ & 90 & 35 & 45 & 3.5 \\
\hline \multicolumn{6}{|c|}{ Recrystallized texture } \\
\hline$\{001\}$ & $\langle 100\rangle$ & $\mathbf{0}$ & 0 & 0 & 6.5 \\
\hline$\{013\}$ & $\langle 100\rangle$ & 0 & 18 & 0 & 4.8 \\
\hline$\{012\}$ & $\langle 100\rangle$ & 0 & 27 & $\mathbf{0}$ & 3.8 \\
\hline$\{011\}$ & $\langle 100\rangle$ & 0 & 45 & 0 & 2.2 \\
\hline
\end{tabular}


suggest that the HB is partially recrystallized and the extent of recrystallization is higher near the surface than that at the centre.

The ODF analysis of the texture components (Figures 2 and 3) near the surface of the HB yields mainly two limited $\langle 110\rangle$ fibre texture components to characterise the two orientation tubes of preferred orientations. One of the fibres (i.e. $\langle 110\rangle \| \mathrm{RD})$ runs from $\{001\}\langle 110\rangle$ to $\approx\{112\}\langle 110\rangle$ with a maximum observed at $\approx\{112\}\langle 110\rangle$. The other fibre (i.e. $\left.\langle 110\rangle 30^{\circ} \mathrm{ND}\right)$ stretches from an orientation $\{111\}\langle 112\rangle+5^{\circ}$ to $\approx\{112\}\langle 110\rangle$. The orientation $\{111\}\langle 112\rangle+5^{\circ}$ is at a distance of $5^{\circ}$ from $\{111\}\langle 112\rangle$ and $3^{\circ}$ from $\left\{\begin{array}{lll}11 & 11 & 8\end{array}\right\}\langle 4 \quad 411\rangle$. Smith (1978) has also observed the components $\{112\}\langle 110\rangle$ and $\{111\}\langle 110\rangle$ in the surface texture of hot rolled austenitic stainless steel, but here, more interestingly, the maximum density is observed at $\left\{\begin{array}{ll}7 & 12\end{array}\right\}\langle 110\rangle$ which is $5^{\circ}$ away from the ideal orientation $\{112\}\langle 110\rangle$, rotated about RD. The other interesting features of the ODF analysis of the surface texture are the observations of two limited fibres i.e. $\langle 110\rangle \| \mathrm{RD}$ and $\langle 110\rangle 30^{\circ} \mathrm{ND}$ fibres. The orientations $\{001\}\langle 110\rangle,\left\{\begin{array}{lll}11 & 11 & 8\end{array}\right\}\langle 4 \quad 411\rangle$ and $\{112\}\langle 110\rangle$ are in fact the ones predicted theoretically as stable orientations by Dillamore and Katoh (1974a) under the action of imposed shear. Further it has been reported that the component $\{111\}\langle 110\rangle$ becomes stable by permitting shear parallel to the rolling plane along TD direction (Dillamore and Katoh, 1974b). In this context it is also important to note that the orientations $\{001\}\langle 110\rangle,\{111\}\langle 110\rangle$ and $\{112\}\langle 110\rangle$ had been observed in 70/30 brass under torsion by Backofen and Hundy (1953) and the orientations $\{001\}\langle 110\rangle$ and $\{111\}\langle 112\rangle$ in $70 / 30$ brass and the orientations $\{001\}\langle 110\rangle,\{111\}\langle 110\rangle,\{112\}\langle 110\rangle$ and $\{111\}\langle 112\rangle$ in $\mathrm{Cu}$ and $\mathrm{Al}$ under shear (Williams, 1962). The presence of a strong $\{112\}\langle 110\rangle$ component on the surface of $\mathrm{Ag}$ under the condition of high friction rolling and high reduction per pass has also been noticed (Regenet and Stüwe, 1963). Recently Truszkowski, Krol and Major (1980) reported $\{001\}\langle 110\rangle,\{111\}\langle 112\rangle$ and $\{111\}\langle 110\rangle$ components in the surface texture of $\mathrm{Al}$ rolled with large deformation per pass, high total reduction in thickness and high friction between metal and rolls and they emphasized the significance of the flow characteristic of the material on the appearance of shear texture (i.e. low yield strength and low work hardening exponent favour the shear texture). Further it has been documented that the shear components $\{001\}\langle 110\rangle$ and $\{111\}\langle$ UVW $\rangle$ develop in face centred cubic metals, the latter being predominant in high SFE materials whereas the shear component $\{112\}\langle 110\rangle$ is predominant in low SFE materials (Dillamore and Roberts, 1965).

The austenitic stainless steel used in the present investigation has low SFE of the order of $38 \mathrm{mJm}^{-2}$ (Rhodes and Thompson, 1977). The ODF analysis of the surface texture of the $\mathrm{HB}$ also indicated a predominant $\approx\{112\}\langle 110\rangle$ component. It has also been reported that the maximum shear strain occurs near the surface of materials (MacGregor and Coffin, 1943, Hansen and Mecking, 1975) depending on the rolling conditions and particularly under the hot rolling conditions. During hot rolling, the flow stress and work hardening exponent are lower as compared with those determined at ambient temperature and also the variable frictional forces within the length of contact of the roll gap produced by the changing speed of a metal between the entry and exit side of the mill will restrict the metal flow near the immediate surface of the metal and thus the maximum shearing strain is produced near the surface of the metal. Further, the actual hot 
rolling conditions in the hot strip mill are far more serious and also there is a decrease in SFE with a decreasing hot rolling temperature (Goodman and $\mathrm{Hu}$, 1964). Thus, all the available information taken together, establish that all the elements of the two limited fibres (i.e. $\langle 110\rangle \| \mathrm{RD}$ and $\langle 110\rangle 30^{\circ} \mathrm{ND}$ ) and also the orientations $\approx\{111\}\langle 110\rangle$ and $\approx\{111\}\langle 123\rangle$ observed in the surface layer of the HB of austenitic stainless steel are shear texture components. The component $\{129\}\left\langle\begin{array}{lll}4 & 11 & 2\rangle\end{array}\right\rangle$ observed in the surface of the HB is associated with recrystallization texture (Musick and Lücke, 1978) and is near to $\{139\}\langle 341\rangle$.

In the hot strip mill, during deformation, the surface layers deform at lower temperature due to the heat losses by conduction to the work rolls whereas the central layers deform at higher temperature due to the heat gain by deformation (Yanagi, 1976). For a $3 \mathrm{~mm}$ thick strip the difference between the temperatures of centre and surface layers is of the order of $373 \mathrm{~K}$. Further during hot rolling, the SFE decreases with decreasing hot rolling temperature. The fact that the finishing temperature of the $\mathrm{HB}$ is about $1173 \mathrm{~K}$, the surface temperature during deformation is lower than that at the centre and the surface layers are partly recrystallized, would suggest that some process of simultaneous shearing and dynamic in situ recrystallization by sub grain growth to a limited extent takes place as the austenitic stainless steel is hot rolled.

On the other hand the ODF (Figure 6) for $S=0$ level of the HB indicates the presence of textural elements of both the retained rolling texture (i.e. copper type) and recrystallization texture (i.e. cube and RD rotated cubes). The fibre $\langle 110\rangle 60^{\circ} \mathrm{ND}$ of retained rolling texture runs from $\{112\}\langle 111\rangle$ through the region of $\approx\{123\}\langle 634\rangle$ to the orientation $\{011\}\langle 112\rangle$ with a maximum at $\{011\}\langle 112\rangle$ position and minimum at $\{112\}\langle 111\}$ position. Further the recrystallized textural elements show a scattering from cube position $\{001\}\langle 100\rangle$ towards Goss position $\{011\}\langle 100\rangle$ along with other elements of RD rotated cubes (i.e. $\{013\}\langle 100\rangle$ and $\{012\}\langle 100\rangle)$. Recently it has been reported that it is the component $\{112\}\langle 111\rangle$ and not the component $\{123\}\langle 634\rangle$ which decreases in favour of cube orientation $\{001\}\langle 100\rangle$ at the onset of recrystallization, even though the cube orientation possesses a good $40^{\circ}\langle 111\rangle$ rotation relationship with the orientation $\{123\}\langle 634\rangle$ (Virnich, Köhlhoff, Lücke et al., 1978. Eichelkraut, Hirsch and Lücke, 1984). Further, the grains of the $\{011\}\langle 112\rangle$ component are consumed by the growth of cube grains but at a much lower rate due to unfavourable orientation relationship (Lücke, 1984). The more rapid disappearance of $\{112\}\langle 111\rangle$ component than the $\approx\{123\}\langle 634\rangle$ component in favour of cube orientation indicates the preferential nucleation of cube grains probably in the transition band developed within $\{112\}\langle 111\rangle$ component. Dillamore and Katoh (1974b) based on oriented nucleation, assumed the presence of nuclei in the divergent zones. These divergent zone orientations are unstable and give rise to formation of transition bands containing the remnant of original orientation during rolling. Further, the orientations in the scattered zone between the cube and Goss positions and also many other orientations having their $\langle 100\rangle$ axis close to ND come under this category. The presence of RD rotated cubes in recrystallization texture has also been interpreted as an evidence of oriented nucleation.

All evidence (i.e. the minima at $\{112\}\langle 111\rangle$ component and the maxima at $\{011\}\langle 112\rangle$ component along with $\approx\{123\}\langle 634\rangle$ as intermediate, all belonging to retained copper type texture, and also the scattering of cube orientation towards 
Goss position $\{011\}\langle 100\rangle$ (all belonging to recrystallization texture) taken together concludes that during hot rolling of austenitic stainless steel, in the hot strip mill, a copper type texture, a characteristic feature of high and intermediate SFE (Goodman and Hu, 1964) develops during deformation. Simultaneously with the development of copper type texture, the dynamic recrystallization by oriented nucleation of divergent zone orientations (Dillamore and Katoh, 1974b) occurs to a limited extent most probably and preferably at the transition bands in the grains of $\{112\}\langle 111\rangle$ orientation. These oriented nuclei grow at the expense of $\{112\}\langle 111\rangle$ component and subsequently at the cost of $\approx\{123\}\langle 634\rangle$ orientation which bears a good $40^{\circ}\langle 111\rangle$ rotation relationship with the cube orientation. However, sufficient time is not available for the growth of the cube grains and other RD rotated cube grains by consuming the grains of remaining orientations $\{112\}\langle 111\rangle, \approx\{123\}\langle 634\rangle$ and $\{011\}\langle 112\rangle$, mainly because of lower finishing temperature $(1173 \mathrm{~K})$ of the $\mathrm{HB}$. This results in the retention of the components of copper type rolling texture and ultimately a partly recrystallized structure is produced at the centre layers of the HB.

\section{CONCLUSIONS}

1. The hot band (HB) of commercially produced austenitic stainless steel is partly recrystallized.

2. The extent of recrystallization is higher near the surface than at the centre.

3. At the surface of the hot band, two limited orientation tubes i.e. a fibre $\langle 110\rangle \| \mathrm{RD}$ axis running from $\{001\}\langle 110\rangle$ to $\approx\{112\}\langle 110\rangle$ and the other fibre $\langle 110\rangle 30^{\circ}$ ND stretching from $\{111\}\langle 112\rangle+5^{\circ}$ to $\approx\{112\}\langle 110\rangle$, with high density near $\{112\}\langle 110\rangle$ are obtained. The orientation elements of these two tubes are formed during hot rolling at the surface by the action of shear which is due to high rolling reduction at elevated temperatures in the hot strip mill. Once these orientations are formed, they get partly recrystallized by dynamic in situ recrystallization.

4. In the centre layer of the hot band a duplex texture consisting of the orientation elements (i.e. $\{112\}\langle 111\rangle, \approx\{123\}\langle 634\rangle$ and $\{011\}\langle 112\rangle$ ) of copper type and the orientation elements (i.e. cube $\{001\}\langle 100\rangle$ and its scatterings towards Goss $\{011\}\langle 100\rangle$ along with $\mathrm{RD}$ rotated cubes $\{013\}\langle 100\rangle$ and $\{012\}\langle 100\rangle$ ) of recrystallization texture is observed. The components of copper type texture are distributed along partial tube of preferred orientations, with $\langle 110\rangle 60^{\circ} \mathrm{ND}$ fibre, running from $\{112\}\langle 111\rangle$ through the region of $\approx\{123\}\langle 634\rangle$ to $\{011\}\langle 112\rangle$ and they are developed during hot rolling. The origin of cube orientation and its scattering towards Goss position, along with RD rotated cubes $\{013\}\langle 100\rangle$ and $\{012\}\langle 100\rangle$, seems to be controlled by oriented nucleation of the orientations of the divergent zones at the transition bands developed in the grains of $\{112\}\langle 111\rangle$ orientation and by oriented growth of cube grains at the expense of $\{112\}\langle 111\rangle$ and $\approx\{123\}\langle 634\rangle$ orientations during concurrent dynamic recrystallization. 


\section{ACKNOWLEDGEMENTS}

The authors would like to express their gratitude to the management of RDCIS, SAIL, Ranchi and to the Head, Dept. of Metallurgy, BHU, Varanasi for according permission to publish this work.

\section{References}

Backofen, W. A. (1950). Trans. Met. Soc. AIME 188, 1454.

Backofen, W. A. and Hundy, B. B. (1953). ibid 197, 61.

Bunge, H. J. (1969). Mathematische Methoden der Texturanalyse, Akademie Verlag, Berlin.

Dillamore, I. L. and Roberts, W. T. (1963/64). J. Inst. Metals 92, 193.

Dillamore, I. L. and Roberts, W. T. (1965). Metal Rev. 10, 271.

Dillamore, I. L. and Katoh, H. (1974a). Metal Sci. 8, 21.

Dillamore, I. L. and Katoh, H. (1974b). ibid 8, 73.

Eichelkraut, H., Hirsch, J. and Lücke, K. (1984). Z. Metallkde. 75, 113.

Gill Sevilano, J., Van Houtte, P. and Aernoudt, E. (1975). Z. Metallkde. 66, 367.

Goodman, S. R. and Hu, H. (1964). Trans. Met. Soc. AIME 230, 1413.

Hansen, J. and Mecking, H. (1975). In Proceedings ICOTOM4: Texture and Properties of Materials, Ed. G. J. Davies, I. L. Dillamore, R. C. Hudd and J. S. Kallend, The Metals Society, Cambridge, 34.

Hibbard, W. R. (1951). Trans. Met. Soc. AIME 191, 1062.

Hirsch, J. and Lücke, K. (1988). Acta Met. 36, 2863.

Kern, R. and Bergmann, H. W. (1978). In Proceedings ICOTOM5: Texture of Materials, Vol. 1, Ed. G. Gottstein and K. Lücke, Springer Verlag, Berlin, 139.

Lücke, K. (1954). Z. Metallkde. 45, 86.

Lücke, K. (1984). In Proceedings ICOTOM7: Texture of Materials, Ed. C. M. Brakman, P. Jongenburger and E. J. Mittemiejer, Netherlands Society of Materials Science, Noordwijkerhout, 195.

MacGregor, C. W. and Coffin, L. F. (1943). J. Appl. Mech. 10, (1) A13.

Musick, R. and Lücke, K. (1978). In Proceedings ICOTOM5: Texture of Materials, Vol. 1, Ed. G. Gottstein and K. Lücke, Springer Verlag, Berlin, 433.

Regenet, P. and Stüwe, H. P. (1963). Z. Metallkde. 54, 273.

Rhodes, C. G. and Thompson, A. W. (1977). Met. Trans. 8A, 1901.

Schulz, L. G. (1949). J. Appl. Phys. 20, 1030.

Smith, T. G. (1978). In Proceedings ICOTOM5: Texture of Materials, Vol. 2, Ed. G. Gottstein and K. Lücke, Springer Verlag, Berlin, 485.

Taylor, G. Y. (1938). J. Inst. Metals 62, 307.

Truszkowski, W., Krol, J. and Major, J. (1980). Met. Trans. 11A, 749.

Van Houtte, P. (1978). In Proceedings ICOTOM5: Texture of Materials, Vol. 1, Ed. G. Gottstein and K. Lücke, Springer Verlag, Berlin, 347.

Van Houtte, P. and Aernoudt, E. (1975). Z. Metallkde. 66, 202.

Van Houtte, P. and Aernoudt, E. (1976). Mat. Sci. Engg. 23, 11.

Virnich, K. H., Köhlhoff, G. D., Lücke, K. and Pospiech, J. (1978). In Proceedings ICOTOM5: Texture of Materials, Vol. 1, Ed. G. Gottstein and K. Lücke, Springer Verlag, Berlin, 475.

Williams, R. (1962). Trans. Met. Soc. AIME 224, 129.

Yanagi, K. (1976). Trans. Iron and Steel Inst. Japan 16, 11. 